\title{
Structural Transition of Bombyx mori Liquid Silk Studied with Vibrational Circular Dichroism Spectroscopy
}

\author{
Toshinori Morisaku, ${ }^{*}$ Sho Arai, ${ }^{*}$ Kohzo Konno, ${ }^{*}$ Yu Suzuki, $* *$ Tetsuo AsaKura, ${ }^{* * *}$ and \\ Hiroharu YUI*广 \\ *Department of Chemistry, Faculty of Science, Tokyo University of Science, 1-3 Kagurazaka, Shinjuku, \\ Tokyo 162-8601, Japan \\ **Tenure-Track Program for Innovative Research, University of Fukui, 3-9-1 Bunkyo, Fukui, Fukui 910-8507, \\ Japan \\ ***Department of Biotechnology, Tokyo University of Agriculture and Technology, 2-24-16 Nakacho, Koganei, \\ Tokyo 184-8588, Japan
}

\begin{abstract}
We investigated the structural transition from liquid silk to silk fibers with vibrational circular dichroism spectroscopy. Liquid silk showed a major right-handed optically active band at around $1650 \mathrm{~cm}^{-1}$ and a minor one at around $1680 \mathrm{~cm}^{-1}$. The former disappeared over time, while the intensity in the latter increased. With the former wavenumber, liquid silk mainly adopted a random-coil structure. In contrast, the latter may reflect an intermediate structure in the transition. Furthermore, two right-handed bands at around 1630 and $1660 \mathrm{~cm}^{-1}$ appeared with the disappearance of the major band, and then the wavenumber of the former shifted to around $1620 \mathrm{~cm}^{-1}$. The shift results from the decrease in the frequency of the CO stretching mode due to the stacking of the $\beta$-sheet that comprises fibers. The band at $1660 \mathrm{~cm}^{-1}$ may reflect another intermediate structure due to its strong correlation with that at $1620 \mathrm{~cm}^{-1}$ in terms of their temporal change in intensity.
\end{abstract}

Keywords Liquid silk, structural transition, intermediate structure, vibrational circular dichroism spectroscopy, Bombyx mori

(Received February 20, 2015; Accepted April 8, 2015; Published August 10, 2015)

\section{Introduction}

Silk fibroin concentrated aqueous solutions that are stored in the middle silk grand of Bombyx mori (B. mori), called liquid silk, are spun out by the pressure applied in the anterior silk gland. ${ }^{1}$ Then, they are quickly converted into insoluble silk fibers. Silk fibers have been applied to the cloth of various industrial products such as clothes and carpets because of their high strength and toughness. ${ }^{2}$ In addition, because silk fibers show excellent biocompatibility, they have been widely used for medical sutures. ${ }^{3}$ Their application to artificial blood vessels is also expected. ${ }^{4}$ Thus, much attention has been focused on the mechanism of the structural transition from liquid silk to silk fibers not only to understand the structural origin of the expression of their excellent mechanical properties and biocompatibility but also to develop materials that are more advanced than silk fibers. ${ }^{5-11}$

For the spectroscopic study of the mechanism, silk fibroin concentrated aqueous solutions that are formed by the lysis of silk fibers in $\mathrm{LiBr}$ or $\mathrm{CaCl}_{2} / \mathrm{C}_{2} \mathrm{H}_{5} \mathrm{OH}$ solutions at high temperatures, called regenerated silk fibroin solutions, have been used. ${ }^{6-9,11}$ By electronic circular dichroism (ECD) and

$\dagger$ To whom correspondence should be addressed.

E-mail: yui@rs.kagu.tus.ac.jp infrared (IR) absorption measurements and X-ray scattering and NMR ones under shear, it was observed that regenerated silk fibroin solutions adopted a random-coil structure and they were converted into crystalline fibers with a Silk II structure, which mainly comprised a $\beta$-sheet structure. ${ }^{6-8,11}$

However, in the studies on the transition to silk fibers, only the regenerated silk fibroin solutions have been used in the initial state of the transition. There is no study where liquid silk is used in the initial state. It has been shown that the secondary structure of regenerated silk fibroin solutions is a random-coil structure. $^{6-8,11}$ In contrast, $\alpha$-helix, random-coil, and repeated type II $\beta$-turn structures have been suggested by ECD, optical rotatory dispersion, and NMR measurements as secondary structures of liquid silk. ${ }^{12-15}$ The secondary structure of liquid silk remains an unsolved issue.

Furthermore, there is a controversy about the existence of intermediates even in the study of the transition from regenerated silk fibroin solutions to silk fibers. Some studies suggested the direct transition from a random-coil structure to a $\beta$-sheet one. ${ }^{6,8,11}$ In contrast, other studies suggested transition models through intermediates. ${ }^{7,9}$ By X-ray scattering under shear and IR absorption measurements, the transition from an amorphous $\beta$-sheet structure to a $\beta$-turn-rich crystalline Silk I one was observed as an intermediate process before the formation of silk fibers. ${ }^{7} \quad$ As another intermediate structure, an $\alpha$-helix-rich crystalline Silk I structure was also observed by X-ray scattering 
(a)

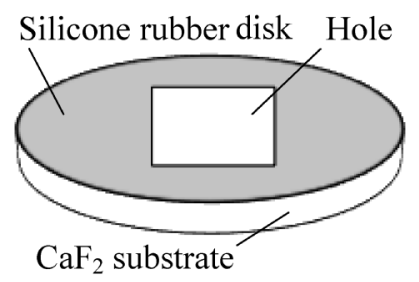

(b)

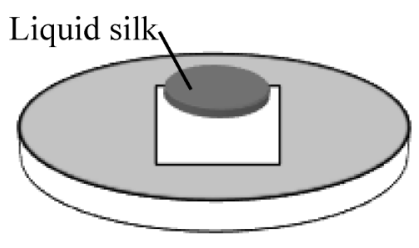

(c)

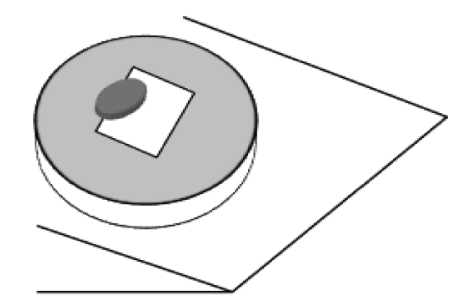

(d)

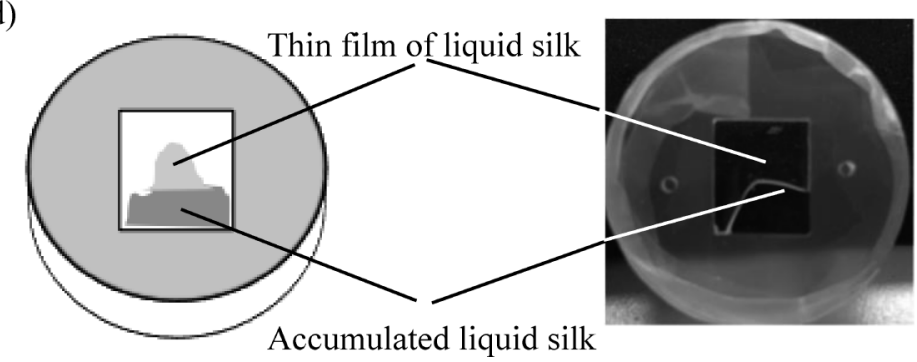

Fig. 1 Schematic illustration of the preparation of the cell. (a) Set of a silicone rubber disk with $10 \mathrm{~mm}$ square hole on a $\mathrm{CaF}_{2}$ substrate. (b) Deposition of the liquid silk on the upper part of the hole. (c) Set of the cell on a tilted surface. (d) Formation of a thin film of liquid silk and image of a prepared cell.

and Raman measurements. ${ }^{9}$ Since liquid silk, not regenerated silk fibroin solutions, is converted into silk fibers, the measurements of its transition are desirable.

Here, we investigated the structural transition of liquid silk with vibrational circular dichroism (VCD) spectroscopy. VCD spectroscopy is a powerful tool for the discrimination of slight differences between secondary structures of proteins in aqueous environments and for the detection of intermediate structures in the denaturation process of proteins. ${ }^{16-19}$ It is worth noting that the present study reported on the structural transition not of regenerated silk fibroin solutions, but of liquid silk. We slowly developed the structural transition of liquid silk by keeping it at room temperature after its extraction from B. mori. From the VCD measurements of the conformational changes in liquid silk, we found that its secondary structure was mainly a randomcoil structure. In addition, we clarified the existence of the intermediate structure that is characterized by the right-handed optically active VCD bands at around 1660 and $1680 \mathrm{~cm}^{-1}$.

\section{Experimental}

\section{Preparation of liquid silk}

The liquid silk used was silk fibroin concentrated solutions extracted from the middle silk gland of B. mori. The structure of air-dried liquid silk, denoted as a Silk I structure, has been widely studied. ${ }^{20-23}$ Recently, liquid silk has been also analyzed by solution NMR methods. ${ }^{15}$ The use of liquid silk is also useful for the discussion with the results by solution NMR methods.

Liquid silk was extracted in the same manner as described previously. ${ }^{15}$ The middle silk glands containing liquid silk were extracted from the B. mori larvae from the sixth to eighth days of the fifth instar. By soaking the glands in a dish filled with fresh distilled water, the liquid silk could be gently removed from the glands. The liquid silk was then soaked more than 5 times in fresh distilled water for 5 min to remove sericin from its surface. ${ }^{24}$ The removal of sericin was confirmed by NMR measurements. The amino-acid composition of silk fibroin from B. mori is Gly $42.9 \%$, Ala $30.0 \%$, Ser $12.2 \%$, Tyr $4.8 \%$, and Val $1.8 \%$, while sericin from $B$. mori is characterized by a high Ser content (approximately 35\%). ${ }^{25,26}$ From NMR measurements, the liquid silk prepared under our experimental condition was composed of Gly $42.9 \%$, Ala $32.1 \%$, Ser $11.6 \%$, Tyr $2.8 \%$, and $1.1 \%$ (data not shown). Because the proportion of Ser in the prepared liquid silk was almost comparable with that of Ser in the literature, we evaluated that sericin was removed from the liquid silk. The concentration of the liquid silk was estimated as $12.5-17.5 \mathrm{w} / \mathrm{w} \%$ from the difference in weight between liquid silk and dried silk. ${ }^{15}$

\section{Preparation of a cell for IR and VCD measurements}

A schematic illustration for the preparation of the cell is shown in Fig. 1. Two $\mathrm{CaF}_{2}$ substrates (diameter: $25.4 \mathrm{~mm}$ ) and a silicone rubber disk (diameter: $25.4 \mathrm{~mm}$, thickness: $3 \mathrm{~mm}$ ) as a spacer were prepared for the cell. There is a hole $(10 \mathrm{~mm}$ square) at the center of the rubber disk. The rubber disk was put on the $\mathrm{CaF}_{2}$ substrate (Fig. 1(a)), then the fresh liquid silk extracted from the $B$. mori was gently deposited to fill up the upper part of the hole (Fig. 1(b)). Next, the deposited liquid silk needs to be thinned to eliminate the strong IR absorption from water. In the preparation of a thin film of the liquid silk, an enforced compression gives it large pressure, leading to the rapid structural transition to silk fibers. To avoid the application of pressure to the liquid silk, we prepared the thin film as follows: the $\mathrm{CaF}_{2}$ substrate where the liquid silk was deposited was slightly tilted (Fig. 1(c)). The liquid silk slowly flowed down the hole. Then, although most of the liquid silk accumulated on the down side of the hole, a small amount of the liquid silk remained thinly adhered on the upper side (Fig. 1(d)). The naturally formed thin film of liquid silk was used for the IR and VCD measurements. The cell was tightly sealed after another $\mathrm{CaF}_{2}$ substrate was put on the silicone rubber disk. 


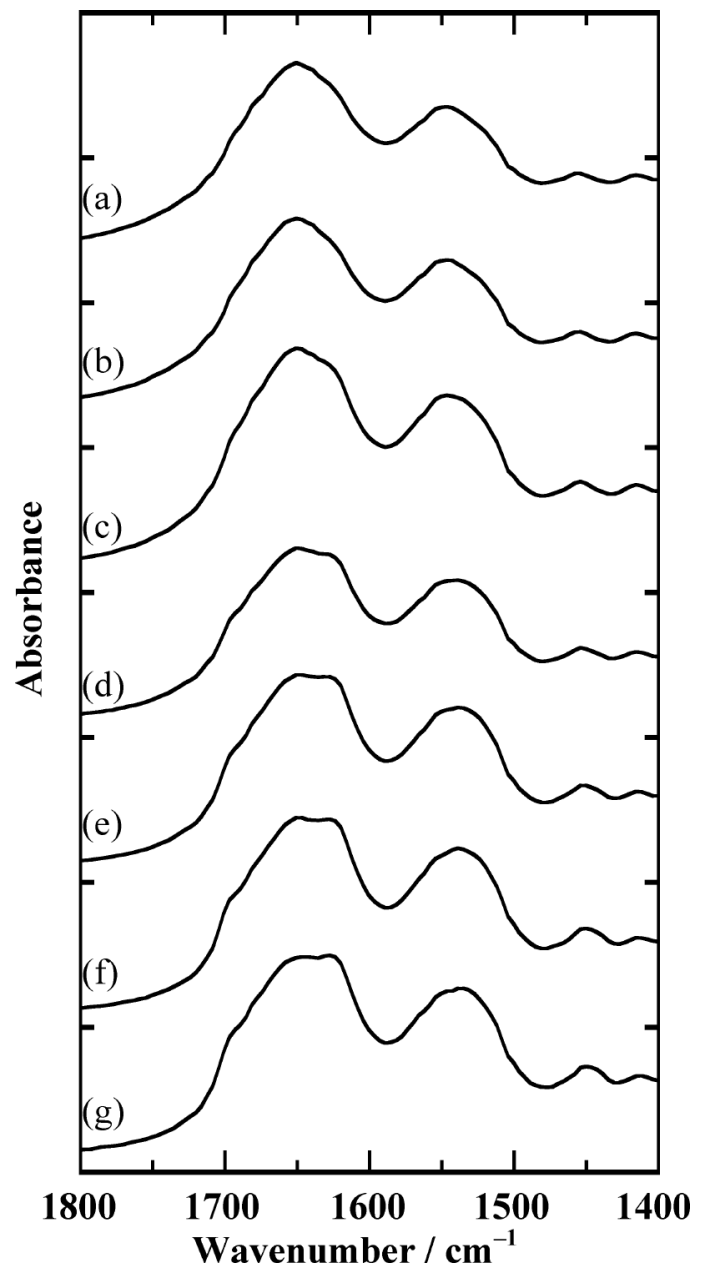

Fig. 2 Temporal change of the IR absorption spectra for the liquid silk in the region $1400-1800 \mathrm{~cm}^{-1}$. Lapsed time from the extraction of the liquid silk: (a) $12 \mathrm{~h}$, (b) $19 \mathrm{~h}$, (c) $29 \mathrm{~h}$, (d) $34 \mathrm{~h}$, (e) $57 \mathrm{~h}$, (f) $120 \mathrm{~h}$, and $(\mathrm{g}) 124 \mathrm{~h}$. One division of the $y$-axis in the IR absorption spectra corresponds to 0.5 absorbance.

\section{IR and VCD measurements}

The detailed instruments for IR and VCD measurements have been previously reported. ${ }^{19}$ Briefly, an IR spectrometer (Nicolet 8700, Thermo Fisher Scientific) directly connected with a tabletop optical module (TOM) box equipped with a photo-elastic modulator (Hinds Instruments, II/ZS50) was used. The prepared cell was set inside the TOM box. The conditions for the measurements were as follows: resolution, $8 \mathrm{~cm}^{-1}$; aperture size, $4.4 \mathrm{~mm}$; and accumulation, 64 times for the IR measurements and 6000 times for the VCD measurements, respectively. All the measurements were performed at $25^{\circ} \mathrm{C}$.

\section{Results and Discussion}

Figures 2 and 3 show the temporal changes of the IR absorption and VCD spectra of the liquid silk in the region $1400-1800 \mathrm{~cm}^{-1}$, respectively. The IR absorption spectra about 12 and $19 \mathrm{~h}$ after its extraction showed an amide I band at $1650 \mathrm{~cm}^{-1}$ and an amide II one at $1548 \mathrm{~cm}^{-1}$ ((a) and (b) in Fig. 2). After about $29 \mathrm{~h}$ from the extraction, two additional shoulder bands at 1625 and $1690 \mathrm{~cm}^{-1}$ were observed in the amide I region ((c) in Fig. 2). The absorbance of both the shoulder bands increased

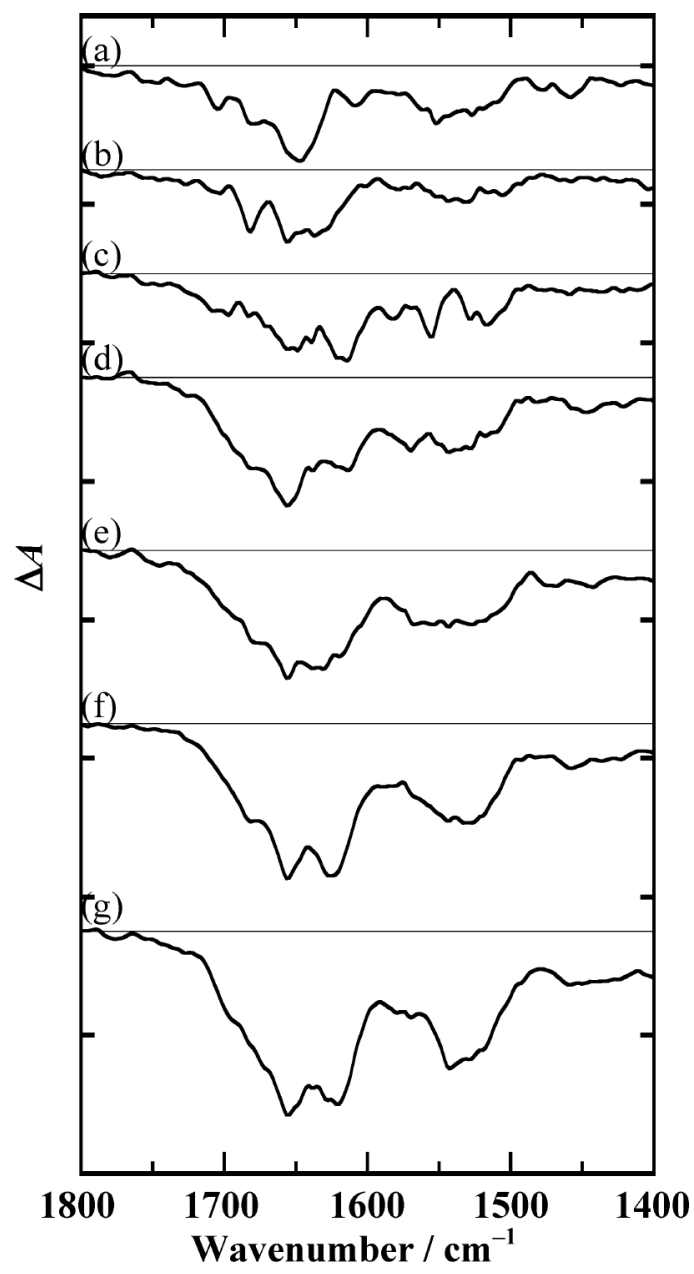

Fig. 3 Temporal change of the VCD spectra for the liquid silk in the region $1400-1800 \mathrm{~cm}^{-1}$. Lapsed time from the extraction of the liquid silk: (a) $12 \mathrm{~h}$, (b) $19 \mathrm{~h}$, (c) $29 \mathrm{~h}$, (d) $34 \mathrm{~h}$, (e) $57 \mathrm{~h}$, (f) $120 \mathrm{~h}$, and (g) $124 \mathrm{~h}$. One division of the $\mathrm{y}$-axis in the VCD spectra corresponds to $2 \times 10^{-4} \Delta A$ unit.

over time ((d) - (g) in Fig. 2).

In the corresponding VCD spectra, we focused on the amide I region, which is the most valuable for the analyses of the secondary structures of proteins. The corresponding VCD spectrum about $12 \mathrm{~h}$ after the extraction showed a major righthanded optically active amide I band at around $1650 \mathrm{~cm}^{-1}$ and a minor right-handed one at around $1680 \mathrm{~cm}^{-1}$ ((a) in Figs. 3 and 4). The former should correspond to the amide I band in the corresponding IR absorption spectrum. By contrast, in the VCD spectrum about $19 \mathrm{~h}$ after the extraction, the major band was not observed but there were two right-handed optically active amide I bands at around 1630 and $1660 \mathrm{~cm}^{-1}$ ((b) in Figs. 3 and 4). The wavenumber $\left(1630 \mathrm{~cm}^{-1}\right)$ of the former shifted to around $1620 \mathrm{~cm}^{-1}$ after about $29 \mathrm{~h}$ from the extraction ((c) in Figs. 3 and 4). In the amide I band at around $1620 \mathrm{~cm}^{-1}$, an increase in intensity and a decrease in width were observed over time ((d) - (g) in Fig. 3). According to the increase in intensity, the intensity of the right-handed optically active amide I bands at around 1660 and $1680 \mathrm{~cm}^{-1}$ also increased ((d- (g) in Fig. 3). The two right-handed optically active amide I bands at around 1620 and $1680 \mathrm{~cm}^{-1}$ should correspond to the two shoulder bands observed in the corresponding IR absorption spectra.

From the temporal changes of the observed spectra, we 


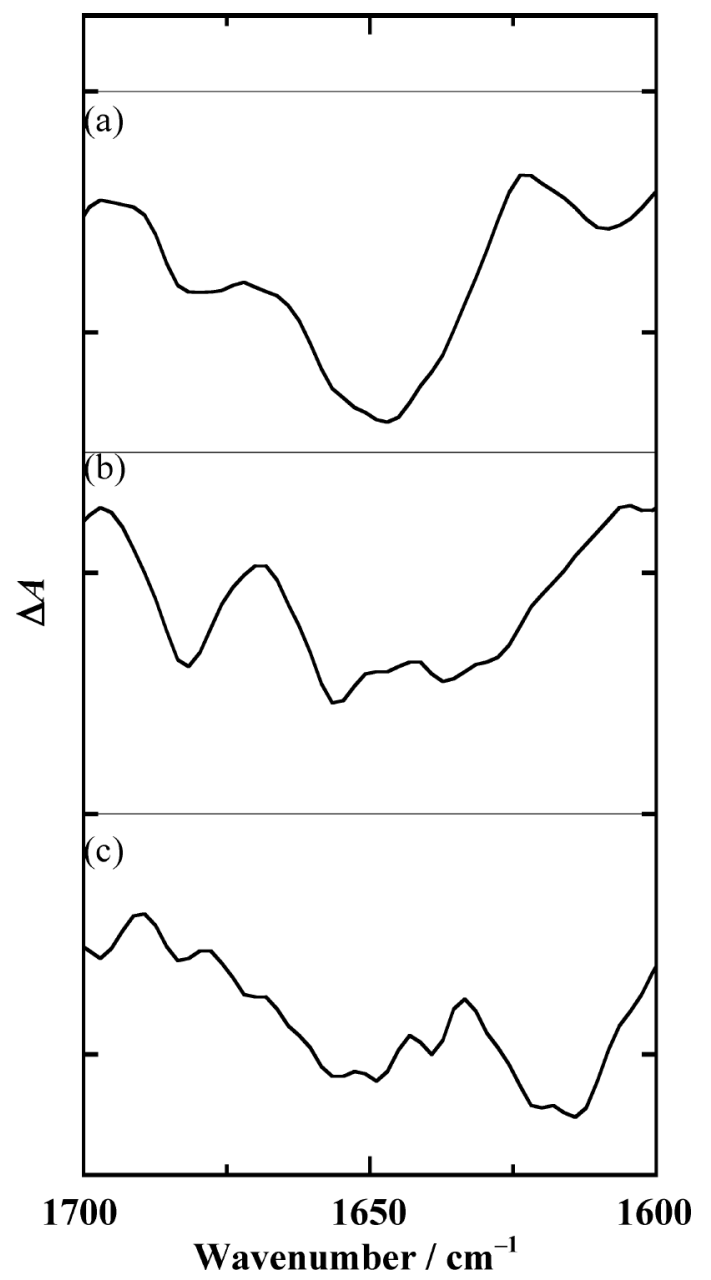

Fig. 4 Enlarged VCD spectra for the liquid silk (a) $12 \mathrm{~h}$, (b) $19 \mathrm{~h}$, and (c) $29 \mathrm{~h}$ after its extraction. One division of the $y$-axis in the VCD spectra corresponds to $1 \times 10^{-4} \Delta A$ unit.

describe the assignment of each band below. The assignment is summarized in Table 1. First, to identify the secondary structure of liquid silk, we characterized the spectrum for silk fibers. From previous spectroscopic studies on the secondary structure of silk fibers, it has been recognized that silk fibers mainly comprise a crystalline Silk II structure, which is mainly composed of a $\beta$-sheet structure..$^{5-11,27-29}$ In general, $\beta$-sheet-rich globular proteins give a negative couplet that comprises a strong right-handed optically active band at around $1630 \mathrm{~cm}^{-1}$ as a main part and a weak left-handed band at around $1660 \mathrm{~cm}^{-1}$ as a minor part. ${ }^{16}$ It has been also reported that the minor band was not observed. ${ }^{17}$ In the VCD spectra under our experimental condition, the main band was clearly observed. Although the minor one was not observed, the former main features showed remarkable similarity to our results. Thus, a right-handed optically active band at around $1630 \mathrm{~cm}^{-1}$, which can be observed about $19 \mathrm{~h}$ after the extraction, can be assigned to a $\beta$-sheet structure, indicating that the fibrillization of the liquid silk already occurred after about $19 \mathrm{~h}$ from its extraction under our experimental conditions.

In addition, the right-handed optically active band at around $1630 \mathrm{~cm}^{-1}$ shifted to around $1620 \mathrm{~cm}^{-1}$ after about $29 \mathrm{~h}$ from the extraction ((c) in Figs. 3 and 4). In the corresponding IR absorption spectrum, the shoulder band at $1625 \mathrm{~cm}^{-1}$ was observed ((c) in Fig. 2). The IR absorption and right-handed
Table 1 Assignment of each VCD band

\begin{tabular}{lcccc}
\hline Wavenumber/cm & 1680 & 1660 & 1650 & $1630 \rightarrow 1620$ \\
\hline Assignment & $\beta$-Turn & $\beta$-Turn & Random-coil & $\beta$-Sheet \\
\hline
\end{tabular}

VCD bands at around $1620 \mathrm{~cm}^{-1}$ have also been observed in fibrils formed from lysozyme and insulin. ${ }^{18}$ The bands are assigned to a stacked $\beta$-sheet structure. ${ }^{18}$ Thus, the shift of the wavenumber from 1630 to $1620 \mathrm{~cm}^{-1}$ is derived from the decrease in the frequency of a $\mathrm{CO}$ stretching vibrational mode due to the stacking of a $\beta$-sheet structure that comprises silk fibers. Interestingly, the shift of the wavenumber may reflect the formation process of silk fibers nucleating a $\beta$-sheet structure as suggested by the formation of amyloid fibrils. ${ }^{30,31}$

It was also observed that the intensity and width of the righthanded band at around $1620 \mathrm{~cm}^{-1}$ increased and decreased over time, respectively $((\mathrm{d})-(\mathrm{g})$ in Fig. 3$)$. The former shows the promotion of the formation of silk fibers, while the latter reflects the formation of more highly crystalline silk fibers over time.

Under our experimental conditions, the liquid silk was transformed into the silk fibers. As the driving force for the transition, we consider the following two factors. One is a shear applied to the liquid silk. It has been well known that a shear effect is a factor for the transition to silk fibers. ${ }^{7,8}$ In the preparation of the thin film of the liquid silk, the deposited liquid silk slowly flowed down the hole of the cell. During the slippage, a shear stress was applied to the liquid silk. The other is a drying of the thin film during the measurements. The drying of water contained in liquid silk leads to the increase in its concentration, resulting in the certain inducement of the structural transition. We evaluated the corresponding changes in the amount of water in the liquid silk from its IR spectra in the $\mathrm{OH}$ stretching vibrational region (Fig. 5(a)). The absorbance of the peaks at $3300 \mathrm{~cm}^{-1}$ decreased over time (Fig. 5(b)), indicating the decrease in the amount of surrounding bulk water.

Next, the secondary structure of the liquid silk is discussed. The VCD band at around $1630 \mathrm{~cm}^{-1}$ shows that a fibrillization of the liquid silk already occurred after about $19 \mathrm{~h}$ from its extraction. In contrast, no band derived from a $\beta$-sheet structure was observed in the VCD spectrum about $12 \mathrm{~h}$ after the extraction, indicating that the spectrum reflected the conformation of the liquid silk ((a) in Figs. 3 and 4). As secondary structures of liquid silk, $\alpha$-helix, random-coil, and repeated type II $\beta$-turn structures have been suggested spectroscopically. ${ }^{12-15}$ In the VCD studies on the secondary structure of hydrated proteins, $\alpha$-helix and random-coil structures have been well-characterized..$^{16,17,19}$ In contrast, with respect to the $\beta$-turn structure, although different model peptides corresponding to the structure have been measured by VCD spectroscopy, there is a controversy about the characteristic wavenumbers and VCD patterns for the $\beta$-turn structure because of their strong dependence on peptide sequences. ${ }^{32-34}$ Thus, we compared the right-handed optically active amide I bands at around 1650 and $1680 \mathrm{~cm}^{-1}$ with the VCD bands that are characteristic of $\alpha$-helix and random-coil structures. ${ }^{16,17,19}$

It has been reported that an $\alpha$-helix structure shows a positive couplet that comprises a strong and narrow right-handed optically active band at around $1660 \mathrm{~cm}^{-1}$ as a main part and a weak left-handed band at around $1640 \mathrm{~cm}^{-1}$ as a minor part. ${ }^{16,17}$ It has been also shown that the minor part was not observed. ${ }^{19}$ The wavenumbers $\left(1650\right.$ and $\left.1680 \mathrm{~cm}^{-1}\right)$ of both the observed bands were different from the main wavenumber of an $\alpha$-helix 

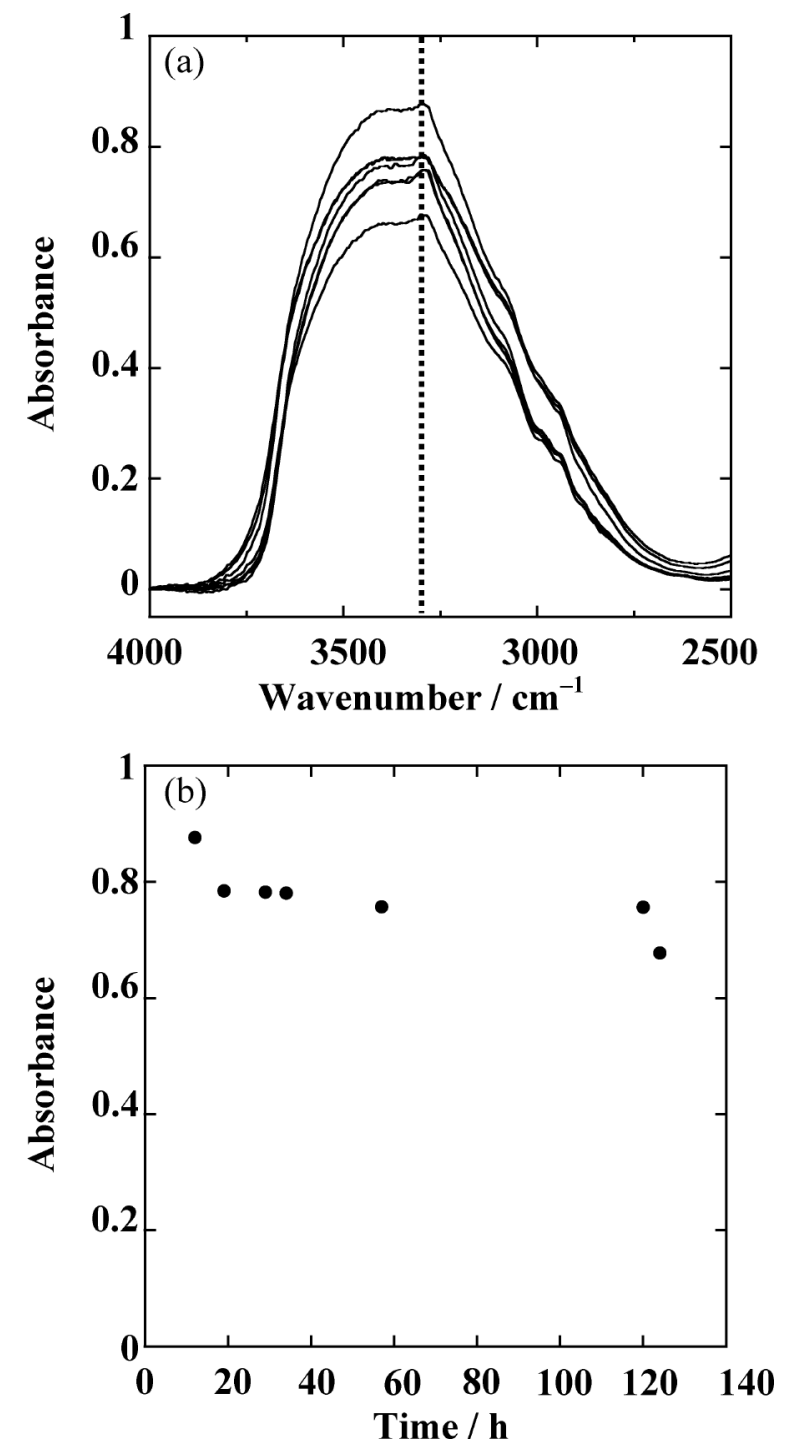

Fig. 5 (a) Temporal change of the IR absorption spectra for the liquid silk in the region $2500-4000 \mathrm{~cm}^{-1}$. The dashed vertical line indicates the position of $3300 \mathrm{~cm}^{-1}$. (b) Time course of the absorbance at $3300 \mathrm{~cm}^{-1}$ in the liquid silk.

structure. Also, the width of their bands was much larger than that of the main right-handed band for an $\alpha$-helix structure. From the wavenumber and width, both the bands at around 1650 and $1680 \mathrm{~cm}^{-1}$ should not be derived from an $\alpha$-helix structure. Next, compared with the VCD band that is characteristic of a random-coil structure, the wavenumber $\left(1650 \mathrm{~cm}^{-1}\right)$ of the major right-handed optically active amide I band was similar to that of casein, which is mainly composed of a random-coil structure. ${ }^{19}$ Although the band of the liquid silk did not broaden toward a lower wavenumber in the amide I region as observed in casein, ${ }^{19}$ the similarity of the wavenumber shows that the major parts of the intermolecular interaction between the $\mathrm{CO}$ and $\mathrm{NH}$ groups through hydrogen bonds in liquid silk sense a similar strength to that of a random-coil structure.

In contrast, the right-handed optically active band at around $1680 \mathrm{~cm}^{-1}$ could not be assigned to the different types of secondary structure that have been characterized by previous VCD studies. Although as secondary structures of liquid silk, $\alpha$-helix, random-coil, and repeated type II $\beta$-turn structures have been suggested, ${ }^{12-15}$ the band could not be derived from $\alpha$-helix and random-coil structures because of its wavenumber. Thus, a repeated type II $\beta$-turn structure may be a candidate. ${ }^{15}$ In addition, because the intensity of the band increased with the promotion of the formation of silk fibers ((d) - (g) in Fig. 2), it is considered that the band reflects not only the structure of the liquid silk but also an intermediate structure in its transition to silk fibers.

Finally, we considered the assignment of the right-handed optically active band at around $1660 \mathrm{~cm}^{-1}$. The intensity of the band increased with the increase in the intensity of the band at around $1620 \mathrm{~cm}^{-1}((\mathrm{~d})-(\mathrm{g})$ in Figs. 3 and 4$)$, indicating that both the bands strongly correlate in terms of their temporal change in intensity. Consequently, the band at around $1660 \mathrm{~cm}^{-1}$ may also reflect another intermediate structure in addition to the band at around $1680 \mathrm{~cm}^{-1}$. It has been well known that an $\alpha$-helix structure gives a right-handed optically active band at around $1660 \mathrm{~cm}^{-1}{ }^{16,17,19}$ In the transition from regenerated silk fibroin solutions to silk fibers, an $\alpha$-helix structure was observed as an intermediate structure. ${ }^{9}$ Then, the content of the $\alpha$-helix structure contained in silk fibers decreased as their formation progressed. Also, there is no report that an $\alpha$-helix structure is contained in the Silk II structure of $B$. mori silk fibers. ${ }^{27-29}$ Hence, it is unlikely that the right-handed optically active band at around $1660 \mathrm{~cm}^{-1}$ is derived from an $\alpha$-helix structure.

The band at around $1660 \mathrm{~cm}^{-1}$ may show a $\beta$-turn structure that is different from the one characterized by the right-handed optically active band at around $1680 \mathrm{~cm}^{-1}$. According to ${ }^{13} \mathrm{C}$ solid-state NMR measurement, a Silk II structure has two types of $\beta$-turn structure. ${ }^{27}$ One is a $\beta$-turn that is contained in the crystalline region that constitutes $55 \%$ of the total silk fiber, the other is a $\beta$-turn in the amorphous Tyr-rich region that makes up the other $45 \%{ }^{27}$ The crystalline region should be formed with the formation of silk fibers. In addition, because the righthanded band at around $1660 \mathrm{~cm}^{-1}$ showed a strong correlation with the band at around $1620 \mathrm{~cm}^{-1}$ in terms of their temporal change in intensity, which is characteristic of a stacked $\beta$-sheet structure, it may be assigned to the $\beta$-turn structure that is contained in the crystalline region. In contrast, the right-handed optically active band at around $1680 \mathrm{~cm}^{-1}$ may show the $\beta$-turn structure in the amorphous region.

\section{Conclusions}

The structural transition from liquid silk to silk fibers was investigated with VCD spectroscopy. Liquid silk showed a major right-handed optically active band at around $1650 \mathrm{~cm}^{-1}$ and a minor one at around $1680 \mathrm{~cm}^{-1}$ in the amide I region. From the former wavenumber, it was shown that liquid silk mainly adopted a random-coil structure. By contrast, the latter band may reflect an intermediate structure in the transition. Furthermore, two right-handed bands at around 1630 and $1660 \mathrm{~cm}^{-1}$ appeared with the disappearance of the band at around $1650 \mathrm{~cm}^{-1}$, and then the wavenumber of the former shifted to $1620 \mathrm{~cm}^{-1}$. The band at around $1620 \mathrm{~cm}^{-1}$ was assigned to a stacked $\beta$-sheet structure that mainly comprised silk fibers. The band at around $1660 \mathrm{~cm}^{-1}$ may reflect another intermediate structure in the transition due to its strong correlation with the band at around $1620 \mathrm{~cm}^{-1}$ in terms of their temporal change in intensity. In the future, with further analysis of the intermediate structure in the transition using computer simulation and an X-ray scattering method, a molecular-level understanding of the formation process of silk fibers and their regulation are expected. 


\section{References}

1. K. S. Hossain, A. Ochi, E. Ooyama, J. Magoshi, and N. Nemoto, Biomacromolecules, 2003, 4, 350.

2. T. Asakura and D. L. Kalpan, "Silk Production and Processing. In Encyclopedia of Agricultural Science", ed. C. J. Arutzen, 1994, Academic Press, New York, 1.

3. G. H. Altman, F. Diaz, C. Jakuba, T. Calabro, R. L. Horan, J. Chen, H. Hu, J. Richmond, and D. L. Kaplan, Biomaterials, 2003, 24, 401.

4. D. Aytemiz, W. Sakiyama, Y. Suzuki, N. Nakaizumi, R. Tanaka, Y. Ogawa, Y. Takagi, Y. Nakazawa, and T. Asakura, Adv. Healthcare Mater., 2013, 2, 361.

5. J. Magoshi, Y. Magoshi, and S. Nakamura, "Mechanism of Fiber Formation of Silkworm. In Silk Polymers", ed. D. Kaplan, W. W. Adams, B. Farmer, and C. Viney, 1994, American Chemical Society, Washington, D.C., 292.

6. Y. Yang, Z. Shao, X. Chen, and P. Zhou, Biomacromolecules, 2004, 5, 773.

7. M. Rössle, P. Panine, V. S. Urban, and C. Riekel, Biopolymers, 2004, 74, 316.

8. K. Ohgo, F. Bagusat, T. Asakura, and U. Scheler, J. Am. Chem. Soc., 2008, 130, 4182.

9. A. Martel, M. Burghammer, R. J. Davies, E. Di Cola, C. Vendrely, and C. Riekel, J. Am. Chem. Soc., 2008, 130, 17070.

10. X. Liu and K.-Q. Zhang, "Silk Fiber-Molecular Formation Mechanism, Structure-Property Relationship and Advanced Applications. In Oligomerization of Chemical and Biological Compounds", ed. C. Lesieur, 2014, InTech, Rijeka, 69.

11. J. Zhong, M. Ma, W. Li, J. Zhou, Z. Yan, and D. He, Biopolymers, 2014, 101, 1181.

12. Y. Kobayashi, T. Fujiwara, Y. Kyogoku, and T. Kataoka, Abstract in the 19th NMR meeting, 1980, 149.

13. T. Kataoka, Y. Kobayashi, T. Fujiwara, and Y. Kyogoku, Abstract in Symposium of study and utilization of nonmulberry silkworms, 1981, 71.

14. T. Asakura, Y. Watanabe, A. Uchida, and H. Minagawa, Macromolecules, 1984, 17, 1075.

15. Y. Suzuki, T. Yamazaki, A. Aoki, H. Shindo, and T. Asakura,
Biomacromolecules, 2014, 15, 104.

16. V. Baumruk and T. A. Keiderling, J. Am. Chem. Soc., 1993, 115, 6939.

17. G. Shanmugam and P. L. Polavalapu, J. Am. Chem. Soc., 2004, 126, 10292.

18. S. Ma, X. Cao, M. Mak, A. Sadik, C. Walkner, T. B. Freedman, I. K. Lednev, R. K. Dukor, and L. A. Nafie, J. Am. Chem. Soc., 2007, 129, 12364.

19. T. Morisaku, S. Arai, and H. Yui, Anal. Sci., 2014, 30, 961.

20. R. D. B. Fraser and T. P. MacRae, "Silks. In Conformation in Fibrous Proteins and Related Synthesic Polypeptides", ed. R. D. B. Fraser and T. P. MacRae, 1973, Academic Press, New York, NY, 293.

21. T, Asakura, J. Ashida, T. Yamane, T. Kameda, Y. Nakazawa, K. Ohgo, and K. Komatsu, J. Mol. Biol., 2001, 306, 291.

22. T. Asakura, K. Ohgo, K. Komatsu, M. Kanenari, and K. Okuyama, Macromolecules, 2005, 38, 7397.

23. T. Asakura, Y. Suzuki, K. Yazawa, A. Aoki, Y. Nishiyama, K. Nishimura, F. Suzuki, and H. Kaji, Macromolecules, 2013, 46, 8046.

24. K. Kataoka and I. Uematsu, Kobunshi Ronbunsyu, 1976, 33, 453.

25. C.-Z. Zhou, F. Confalonieri, M. Jacquet, R. Perasso, Z.-G. Li, and J. Janin, Proteins: Struct., Funct., Genet., 2001, 44, 119.

26. Y. Takasu, H. Yamada, and K. Tsubochi, Biosci. Biotechnol. Biochem., 2002, 66, 2715.

27. T. Asakura, J. Yao, T. Yamane, K. Umemura, and A. S. Ulrich, J. Am. Chem. Soc., 2002, 124, 8794.

28. R. Valluzzi and H.-J. Jin, Biomacromolecules, 2004, 5, 696.

29. S. Ling, Z. Qi, D. P. Knight, Z. Shao, and Z. Chen, Biomacromolecules, 2011, 12, 3344.

30. J. M. Kenney, D. Knight, M. J. Wise, and F. Vollrath, Eur. J. Biochem., 2002, 269, 4159.

31. U. Slotta, S. Hess, K. Spiess, T. Stromer, L. Serpell, and T. Scheibel, Macromol. Biosci., 2007, 7, 183.

32. P. Xie, Q. Zhou, and M. Diem, J. Am. Chem. Soc., 1995, 117, 9502.

33. J. Hilario, J. Kubelka, and T. A. Keiderling, J. Am. Chem. Soc., 2003, 125, 7562.

34. A. Borics, R. F. Murphy, and S. Lovas, Biopolymers, 2007, 85,1 . 